Quebecor - Position Product Application Focus graphic and blurb here. Pick up from Vol. 11, No. 3, p. 392

\title{
Multiplex Detection and Quantitation of Proteins on Western Blots Using Fluorescent Probes
}

\author{
Jeffrey C. Gingrich, Diana R. Davis and Quan Nguyen \\ Bio-Rad Laboratories, Hercules, CA, USA
}

BioTechniques 29:636-642 (September 2000)

\begin{abstract}
The uses of multiplex detection methodologies are dramatically increasing as a means to increase sample throughput and to demonstrate quantitative differences between multiple targets in gene or protein expression analysis. In this study, we investigate the application of multiplex fluorescent detection for three proteins on the same Western blot using a laser-scanning imaging system, the Bio-Rad Molecular Imager ${ }^{\circledR} F X$. We show that independent detection and quantitation of multiple targets is achievable with little or no correction for fluorescent crosstalk by using fluorescent tags preferentially excited with different laser lines and detected at wavelengths that minimize fluorescence crosstalk. We demonstrate that the use offluorescent detection methods can provide a tenfold greater quantifiable range but with two- to fourfold less sensitivity than chemiluminescent detection methodologies. Two examples of three-color multiplex detection using FITC-, Cy ${ }^{\mathrm{TM}} 3$ - and Cy $5^{\mathrm{TM}}$-conjugated probes on Western blots are provided to demonstrate applications of this approach.
\end{abstract}

\section{INTRODUCTION}

With the rapid increase in the collection of DNA and gene sequences from numerous organisms laying the foundation for the study of "functional genomics", there is now greater interest in understanding multiple or global gene expression in biological systems. Multiplexing is commonly used in genetic analysis in applications that range from automated DNA sequencing to DNA or gene expression "chips" to chromosome painting. In protein analysis, confocal microscopy uses multiplex detection to demonstrate the relative localization of different proteins in cells.

Despite such widespread uses of multiplex systems, the most common method for analyzing protein expression levels is Western blotting with detection of a single specific protein target using horseradish peroxidase- or alkaline phosphataseconjugated antibody probes combined with colorimetric or chemiluminescent detection $(1,2,6,14)$. While these methods are sufficient for studying a single target, they are unsuitable for analyzing multiple targets at the same time, particularly if the targets are of unknown or of similar size. To analyze multiple targets, the blot is typically stripped and reprobed for additional targets of interest (4). While reprobing does take additional time, there are other reasons not to reprobe a blot multiple times. Often, some of the target protein on the blot is removed as a result of the probe stripping procedure. If one protein is removed to a greater or lesser extent relative to another protein, the ability to quantitate the relative amounts of different proteins of interest is lost.

In one approach demonstrating multiplex Western blotting, multiple protein targets have been distinguished using multiple colorimetric indicators, either alone (11) or in combination with chemiluminescent detection (13). However, this approach does not translate well to a film or digitally captured imaging because colorimetric indicators are generally not well discrim inated by optical filters. Furthermore, one of the colorimetric indicators that has been used (immunogold/silver staining) results in colors ranging from yellow, orange, red to brown in color, thus making it all the more difficult to discriminate colorimetric indicators by specific optical filters (11). 
Only recently has the use of fluorescent probes been reported for Western blot analysis $(3,8)$. Results of two-color detection have only been briefly mentioned using a single laser system, with the caveat that additional optical filters and software corrections would be necessary to separately distinguish the two probe colors (8). To multiplex proteins on a single Western blot, each probe is conjugated with a different fluorochrome with emission spectra at wavelengths distinguishable from the other probes. Using different excitation wavelengths with different laser excitation sources and the appropriate selection of emission filters, the need for software correction for fluorescence overlap between the different fluorochromes (as typically found when exciting with a single laser line) can be virtually eliminated.

Here, we present studies to demonstrate independent three-color detection of proteins on Western blots using a three-laser scanning imager. The linear range and limit of detection are compared to chemiluminescent detection. Two model systems are then used to demonstrate independent detection of three proteins on Western blots.

\section{MATERIALS AND METHODS}

\section{Proteins and Antibodies}

Human apo-transferrin was purchased from Sigma (St. Louis, MO, USA). Human peripheral blood serum samples were a gift from Bio-Rad Immunodiagnostics Division (Hercules, CA, USA). Rabbit anti-human transferrin and mouse anti-human IgG were purchased from Dako (Carpenteria, CA, USA). Mouse anti-human caspase 8 was purchased from BD PharMingen (San Diego, CA, USA), and goat anti-human albumin was purchased from Sigma. Rabbit anti-human PKA $\alpha$ cat (PKA) and goat anti-human actin were purchased from Santa Cruz Biotechnology (Santa Cruz, CA, USA).

Donkey secondary antibodies (FITC-conjugated anti-goat, Cy3-conjugated anti-mouse and Cy5-conjugated anti-rabbit) as well as FITC-, Cy3- and Cy5-conjugated goat anti-rabbit secondary antibodies were purchased from Jackson ImmunoResearch Laboratories (West Grove, PA, USA). All fluorescent antibodies were resuspended at $1 \mathrm{mg} / \mathrm{mL}$ in $50 \%$ glycerol and stored protected from light at $-20^{\circ} \mathrm{C}$. Goat antirabbit horseradish peroxidase-conjugated secondary antibody was obtained from Bio-Rad Laboratories.

\section{Culture and Stimulation of Jurkat and HeLa Cells}

Jurkat and HeLa cell lines were obtained from ATCC (Manassas, VA, USA). Jurkat cells were maintained at a concentration of $1 \times 10^{6}$ cells/mL in RPMI-1640 medium supplemented with $10 \%$ fetal bovine serum (FBS), $10 \mathrm{U} / \mathrm{mL}$ penicillin and $10 \mu \mathrm{g} / \mathrm{mL}$ streptomycin (Life Technologies, Rockville, MD, USA). HeLa cells were maintained at approximately $60 \%$ confluency in T75 tissue culture flasks in Ham's F-12 nutrient medium supplemented with $10 \% \mathrm{FBS}, 10 \mathrm{U} / \mathrm{mL}$ penicillin and $10 \mu \mathrm{g} / \mathrm{mL}$ streptomycin. Each cell line was stimulated to undergo apoptosis by the addition of $1 \mu \mathrm{M}$ dexamethasone (Sigma) for $24 \mathrm{~h}$ at $37^{\circ} \mathrm{C}$ and $5 \% \mathrm{CO}_{2}$. An unstimulated control population was included for each cell line. Following stimulation, HeLa cells were detached from T75 flasks by the addition of $100 \mu \mathrm{L} 0.5 \%$ trypsin and $5.3 \mathrm{mM}$
EDTA for $3 \mathrm{~min}$. The cells were washed twice with sterile PBS following detachment (12). Jurkat cells were harvested from T75 flasks and washed twice in PBS. Approximately $5 \times$ $10^{7}$ cells per sample were transferred to $500-\mu \mathrm{L}$ microcentrifuge tubes. The samples were then centrifuged at $1000 \times g$ for $4 \mathrm{~min}$, and the PBS was decanted before cell lysis.

\section{Preparation of Cell Lysates}

Cell lysates were prepared using RIPA buffer $(1 \times$ PBS, $1 \%$ Nonidet $^{\mathrm{TM}} \mathrm{P}-40,0.5 \%$ sodium deoxycholate and $1 \%$ SDS). Protease inhibitors were added to the RIPA buffer in the following concentrations: $100 \mu \mathrm{g} / \mathrm{mL}$ PMSF in isopropanol, 10 $\mu \mathrm{g} / \mathrm{mL}$ aprotinin, $10 \mu \mathrm{g} / \mathrm{mL}$ Pepstatin A and $20 \mu \mathrm{g} / \mathrm{mL}$ Leupeptin (all from Sigma). A volume of $250 \mu \mathrm{L}$ ice-cold RIPA buffer containing freshly added inhibitors was added to each cell pellet. Tubes were carefully mixed with a pipettor and incubated on ice for $30 \mathrm{~min}$. Following incubation, cells were passed through a syringe fitted with a $16-\mathrm{Ga}$ needle several times. Additional PMSF was added $(100 \mu \mathrm{g} / \mathrm{mL})$, and lysates were incubated on ice for $30 \mathrm{~min}$. Cell lysates were centrifuged at $15000 \times g$ for $30 \mathrm{~min}$ at $4^{\circ} \mathrm{C}$ and transferred to clean microcentrifuge tubes. Lysates were stored at $-70^{\circ} \mathrm{C}$ until analysis.

\section{Electrophoresis and Blotting of Protein Samples}

Protein samples were electrophoresed at $100 \mathrm{~V}$ for $90 \mathrm{~min}$ using Tris-HCl, 10-well 12\% acrylamide Ready Gels (BioRad Laboratories). Proteins were electroblotted to supported nitrocellulose (Bio-Rad Laboratories) using a Mini Trans-Blot Cell as described by the manufacturer (Bio-Rad Laboratories).

For the production of dot blots, a twofold serial dilution of human transferrin was generated in PBS beginning at 32 $\mathrm{ng} / \mu \mathrm{L}$. One microliter of diluted protein was spotted directly onto nitrocellulose membrane strips using a micropipettor, and the spots were air dried before processing.

\section{Western Blot Processing and Analysis}

Protein blots were blocked for $1 \mathrm{~h}$ in PBST (PBS $+0.2 \%$ Tween $\left.{ }^{\circledR} 20\right)$ containing 5\% Blotting Grade Blocker Non-Fat Dry Milk (Bio-Rad Laboratories), followed by $3 \times 5$ min washes in PBST. The blots were then incubated for $1 \mathrm{~h}$ in primary antibody (1:1000 dilution in PBST containing $1 \%$ Blocker) at room temperature, washed again $3 \times 5 \mathrm{~min}$ in PBST and incubated for $1 \mathrm{~h}$ in secondary antibody (1:500 dilution in PBST containing 1\% Blocker).

Following $3 \times 5$ min washes in PBST and a single wash for 5 min with PBS, the blots were imaged using a Molecular Im ager FX (Bio-Rad Laboratories) with three lasers (532-nm internal Nd:YAG, 488-nm external Ar ion and 635-nm external red diode laser). FITC-labeled samples were imaged using the 488-nm laser with the standard $530 \mathrm{~nm} \pm 30 \mathrm{~nm}$ bandpass emission filter (530DF30; Omega Optical, Brattleboro, VT, USA). The Cy3-labeled samples were imaged using the internal 532-nm laser with a $605 \mathrm{~nm} \pm 50 \mathrm{~nm}$ bandpass emission filter (605DF50; Omega Optical). The 605-nm bandpass emission filter replaces a 550 longpass emission filter for visualizing $\mathrm{Cy} 3$ independent of $\mathrm{Cy} 5$ when the $\mathrm{FX}$ instrument includes a 635-nm laser. The Cy5-labeled samples were imaged using the external 635-nm laser with the standard $695 \mathrm{~nm} \pm 55 \mathrm{~nm}$ bandpass emission filter (695DF55; Omega Optical). 
Chemiluminescent samples were processed identically to the fluorescent samples. ECL ${ }^{\circledR}$ Western blotting detection reagents were purchased from Amersham Pharmacia Biotech (Piscataway, NJ, USA). Images were captured either on XOmat $^{\mathrm{TM}}$ AR film (Eastman Kodak, Rochester, NY, USA) or by using the Fluor-S ${ }^{\circledR}$ MAX MultiImager (Bio-Rad Laboratories) and analyzed using the Quantity One ${ }^{\circledR}$ version 4.1 software package (Bio-Rad Laboratories). For the three-color images, the three independent images were exported from Quantity One as 8-bit TIFF files and merged using Confocal Assistant (Bio-Rad Laboratories) software.

\section{RESULTS}

\section{Optimization of Three-Color Detection}

For initial characterization of independent three-color detection, we chose a set of antibodies conjugated with one of three fluorochromes whose fluorescence excitation peaks corresponded to three laser lines available on the Bio-Rad Molecular Imager FX laser scanning imager: FITC, Cy3 and Cy5. Imaging of FITC independent from $\mathrm{Cy} 3$ and $\mathrm{Cy} 5$ was achieved using a 488-nm laser with a 530-nm bandpass emission filter. When using the 530-nm bandpass filter-the standard filter supplied for use with the 488-nm laser-there was a small amount of fluorescence emission seen from $\mathrm{Cy} 3$ (about $3 \%$ of the FITC signal when comparing equal amounts of protein) (Table 1 and Figure 1). For better discrimination of FITC from $\mathrm{Cy} 3$, the overlap from $\mathrm{Cy} 3$ could be decreased to less than $0.1 \%$ by using a $510-\mathrm{nm}$ bandpass filter in place of the standard 530-nm bandpass filter. However, this discrimination occurs at the expense of about a $50 \%$ decrease in signal from FITC. Figure 1 and Table 1 show results using the standard 530-nm bandpass filter.

The imaging of $\mathrm{Cy} 3$ probes independent from FITC and Cy5 was achieved with 532-nm laser excitation using a 605$\mathrm{nm}$ bandpass emission filter. With the use of this laser/emission filter combination, there are $0.5 \%$ and $0.3 \%$ of the $\mathrm{Cy} 3$ signal visible from FITC and Cy5, respectively (Table 1 and Figure 1). This 605-nm bandpass emission filter appears optimal for distinguishing $\mathrm{Cy} 3$ from FITC and Cy5 because movement of this bandpass filter to shorter or longer wavelengths resulted in more interference from spectral overlap from either FITC or Cy5 (data not shown).

Imaging of Cy5 independently of $\mathrm{Cy} 3$ and FITC was achieved with a 635-nm laser using a 695-nm bandpass emission filter. With this laser/filter combination, there is less than $0.1 \%$ of the Cy 5 signal intensity from either $\mathrm{Cy} 3$ or FITC (Table 1 and Figure 1).

\section{Linearity and Limit of Fluorescent Compared with Chemiluminescent Detection}

For initial studies on the linearity and limit of fluorescent detection of proteins on Western blots, we used blots in which a protein (human apo-transferrin) was spotted directly onto the filter membrane. The direct spotting approach bypasses any variability in the transfer of proteins from a gel and allows for a more quantitative comparison of the fluorescence detection methods and chemiluminescent detection. Equal volumes of a twofold serial dilution of transferrin protein con-
Table 1. Flourescence Levels

\begin{tabular}{|lcccc|}
\hline & & \multicolumn{3}{c|}{$\begin{array}{c}\text { Relative Level of } \\
\text { Fluorescence (\%) }\end{array}$} \\
\cline { 3 - 5 } $\begin{array}{l}\text { Laser } \\
\text { (nm) }\end{array}$ & $\begin{array}{c}\text { Emission Filter } \\
\text { (bandpass) }\end{array}$ & FITC & Cy3 & Cy5 \\
\hline 488 & 530 & 100 & 3 & $<0.1$ \\
532 & 605 & 0.5 & 100 & 0.3 \\
635 & 695 & $<0.1$ & $<0.1$ & 100 \\
Relative fluorescent signal from equivalent amounts of pro- \\
tein (2 ng protein, spot 5 on Figure 1) that was hybridized \\
with probes conjugated with the indicated fluorochrome. \\
\hline
\end{tabular}

taining from $1 \mu \mathrm{g}$ to as little as $1 \mathrm{pg}$ were spotted directly onto nitrocellulose membranes. The filters were then processed equivalently, first with rabbit anti-human antibody, followed by a secondary fluorescently labeled or HRP-conjugated goat anti-rabbit antibody. Fluorescent detection used the Molecular Imager FX laser scanner and chemiluminescence detection was achieved using both a Bio-Rad Fluor-S MAX MultiImager (a CCD-based imager) and film (Figure 1). Plotting the fluorescent and chemiluminescent signal counts relative to the amount of protein spotted demonstrated that the signal is linear up to $16 \mathrm{ng}$ spotted protein. This is approximately a tenfold higher protein concentration compared to chemiluminescent detection on the Fluor-S MAX MultiImager system (Figure 2 and Table 2).

The limit of detection of transferrin protein directly spotted in the membrane was 2-4 pg using fluorescent detection compared to $1 \mathrm{pg}$ using chemiluminescence detection (Figure 1 and Table 2). When the proteins are blotted from a gel instead of spotted directly on the membrane, the limit of detection using fluorescent detection is $250-500 \mathrm{pg}$ versus $125 \mathrm{pg}$ using chemiluminescent detection (Figure 3 and Table 2). Therefore, with the use of either the direct spotting method or gel transfer, the two- to fourfold difference in limit of detection between fluorescent and chemiluminescent detection remains the same.

\section{Independent Fluorescent Detection of Three Proteins on Western blots}

We next wanted to demonstrate multiplex detection using samples containing a complex mixture of proteins. One approach to demonstrate detection of multiple proteins is to use primary antibodies conjugated directly with different fluorochromes. However, the desired primary antibodies conjugated with three different fluorochromes were not commercially available for the test systems we chose. Therefore, we used non-fluorescent primary antibodies raised in three different species: goat, mouse and rabbit. Donkey secondary antibodies conjugated with FITC, Cy3 and Cy5 were then obtained and directed against the goat, mouse and rabbit antibodies. The antibody sets used for the multiplex detection models described below are listed in Table 3.

In the first example of three-color detection, whole cell lysates were prepared from two cultured cell lines before and after apoptosis induction. Cell lysates prepared from equal 
Table 2. Range and Detection Limits of Fluorescent versus Chemiluminescent Detection

\begin{tabular}{|c|c|c|c|}
\hline \multirow[b]{2}{*}{ Detection Method } & \multirow{2}{*}{$\frac{\text { Linear Rangea }^{\mathrm{a}}}{\text { Dot Format }}$} & \multicolumn{2}{|c|}{ Limit of Detection b } \\
\hline & & Dot format & Gel format \\
\hline \multicolumn{4}{|l|}{ Fluorescent: } \\
\hline FITC & $8 \mathrm{pg}$ to $16 \mathrm{ng}$ & $4 \mathrm{pg}$ & $500 \mathrm{pg}$ \\
\hline Cy3 & $8 \mathrm{pg}$ to $16 \mathrm{ng}$ & $4 \mathrm{pg}$ & $500 \mathrm{pg}$ \\
\hline Cy5 & $4 \mathrm{pg}$ to $16 \mathrm{ng}$ & $2 \mathrm{pg}$ & $250 \mathrm{pg}$ \\
\hline \multicolumn{4}{|l|}{ Chemiluminescent: } \\
\hline ECL- FS MAX & $8 \mathrm{pg}$ to $2 \mathrm{ng}$ & $1 \mathrm{pg}$ & $125 \mathrm{pg}$ \\
\hline ECL-Film & $15 \mathrm{pg}$ to $500 \mathrm{pg}$ & $1 \mathrm{pg}$ & $125 \mathrm{pg}$ \\
\hline $\begin{array}{l}\text { a Linear range }=\mathrm{P}^{2} \\
\text { bLimit of detection }=\end{array}$ & $\begin{array}{l}.95 \\
\text { ound signal }+2 \mathrm{SD}\end{array}$ & & \\
\hline
\end{tabular}

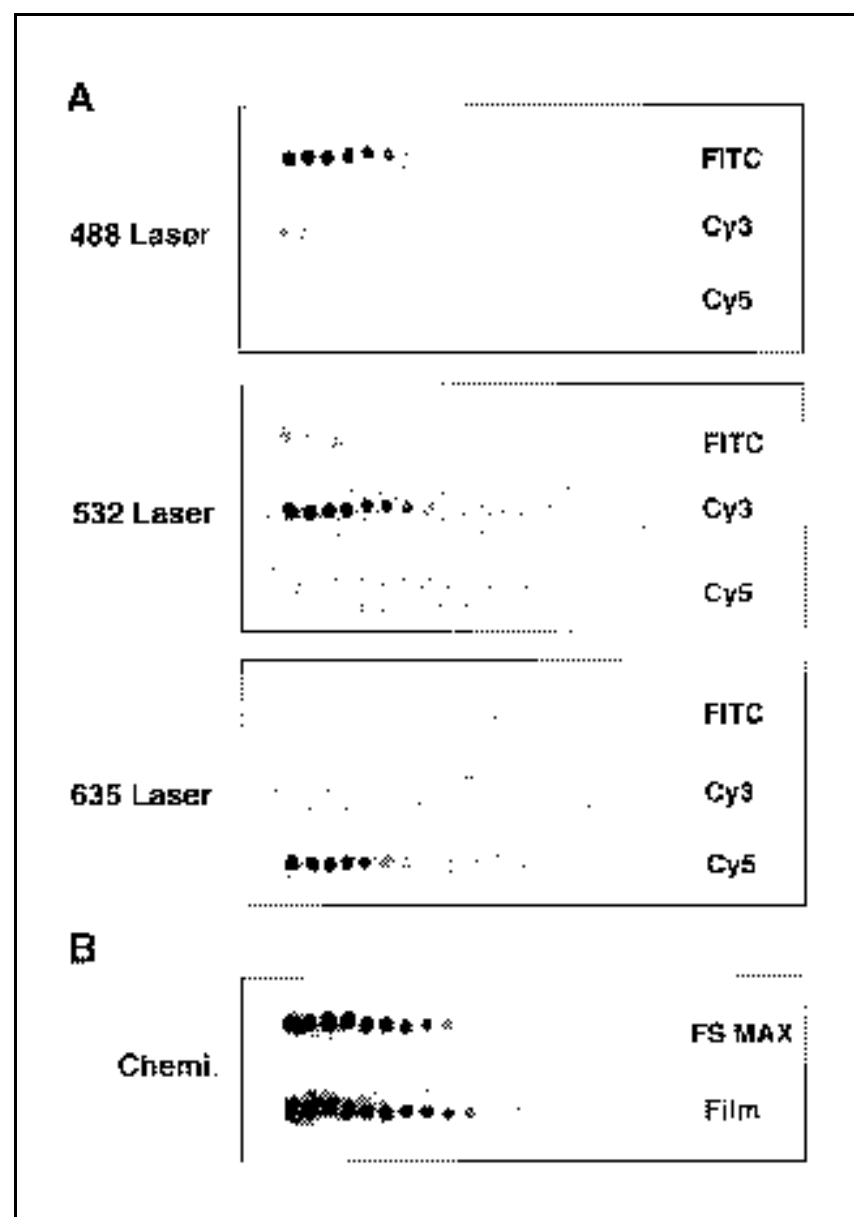

Figure 1. Detection of transferrin protein. (A) Three nitrocellulose mem branes were spotted with $1 \mu \mathrm{L}$ of a twofold serial dilution of human apotransferrin protein starting at $32 \mathrm{ng}$ protein. All membranes were processed first with rabbit anti-human antibody, followed by goat anti-rabbit secondary antibody labeled with either FITC (top membranes), Cy3 (middle mem branes) or Cy5 (bottom membranes). Each set of three blots was scanned on the Bio-Rad Molecular Imager FX using either a 488-nm laser to selectively excite FITC (top membrane set), a 532-nm laser to selectively excite Cy3 (middle membrane set) or a 635-nm laser to selectively excite Cy5 (bottom membrane set). (B) The same serial dilution of transferrin protein shown in (A) was processed using HRP-conjugated secondary antibody and incubated with ECL to generate chemiluminescence. The blots were imaged for $1 \mathrm{~min}$ with a CCD imaging system (FS MAX, Bio-Rad Fluor-S MAX), followed immediately by exposure to X-ray film (Film) for $1 \mathrm{~min}$. numbers of cells were electrophoresed on an SDS-polyacrylamide gel. Following blotting, the membrane was probed simultaneously for three proteins, caspase 8, actin and PKA. Caspase 8 has been shown to be up-regulated during apoptosis (9), while actin and PKA are not expected to change in relative abundance. As shown in Figure 4, actin and PKA appear similar in relative abundance before and after apoptosis induction. Quantitation of the ratio of the fluorescent signal from PKA compared to actin in the cell lysates before and after apoptosis induction shows that PKA increases 1.3 and 1.1 times relative to actin in Jurkat and HeLa cells, respectively. In contrast, the visual intensities of fluorescence seen in Figure 4 indicate that caspase 8 is clearly up-regulated following dexamethasone treatment. In Jurkat cells, the fluorescent signal from caspase 8 increases 4.4 times relative to actin and 3.4 times relative to PKA after apoptosis induction. In HeLa cells, the fluorescence

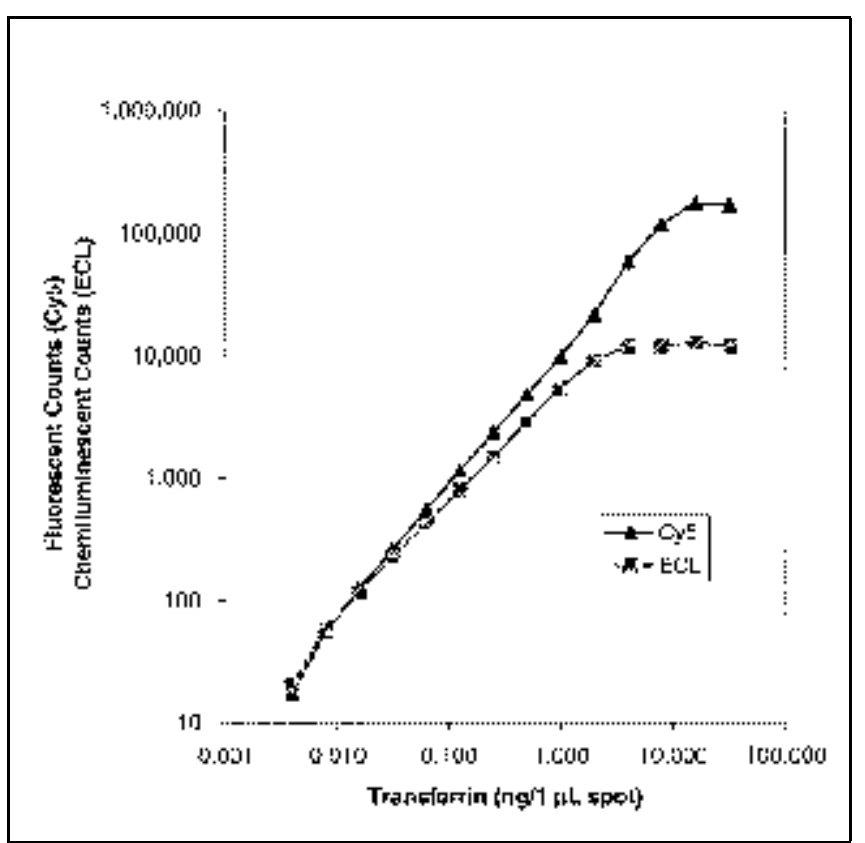

Figure 2. Linearity of detection using fluorescent (Cy5) compared to chemiluminescent (ECL) detection. Signal from the serial dilution of proteins shown in Figure 1 was corrected for background and plotted versus the amount of protein spotted. The signal was linear up to $2 \mathrm{ng}$ with chemiluminescence and up to $20 \mathrm{ng}$ with fluorescent detection. 


\begin{tabular}{|c|c|c|c|}
\hline \multicolumn{4}{|l|}{ Probe Set 1} \\
\hline Protein probed & Actin & Caspase 8 & PKA \\
\hline Reporter dye & FITC & Су3 & Cy5 \\
\hline Primary Ab & GAH-actin & MAH-caspase & RAH-PKA \\
\hline Secondary Ab & FITC-DAG & Сy3-DAM & Cy5-DAR \\
\hline \multicolumn{4}{|l|}{ Probe Set 2} \\
\hline Protein probed & Albumin & $\lg G$ & Transferrin \\
\hline Reporter dye & FITC & Cy3 & Cy5 \\
\hline Primary Ab & GAH-albumin & MAH-IgG & RAH-transferrin \\
\hline Secondary Ab & FITC-DAG & Cy3-DAM & Cy5-DAR \\
\hline \multicolumn{4}{|l|}{ Key: } \\
\hline GAH: Goat anti-human & \multicolumn{3}{|c|}{ DAG: Donkey anti-goat } \\
\hline MAH: Mouse anti-human & \multicolumn{3}{|c|}{ DAM: Donkey anti-mouse } \\
\hline RAH: Rat anti-human & \multicolumn{3}{|c|}{ DAR: Donkey anti-rabbit } \\
\hline
\end{tabular}

signal from caspase 8 increases less than in HeLa cells but is still increased 1.7 times relative to actin and 1.6 times relative to PKA after apoptosis induction.

In the second example of three-color detection, equal amounts of human serum from three patients were electrophoresed on an SDS-polyacrylamide gel. The proteins were subsequently blotted and probed with antibodies against

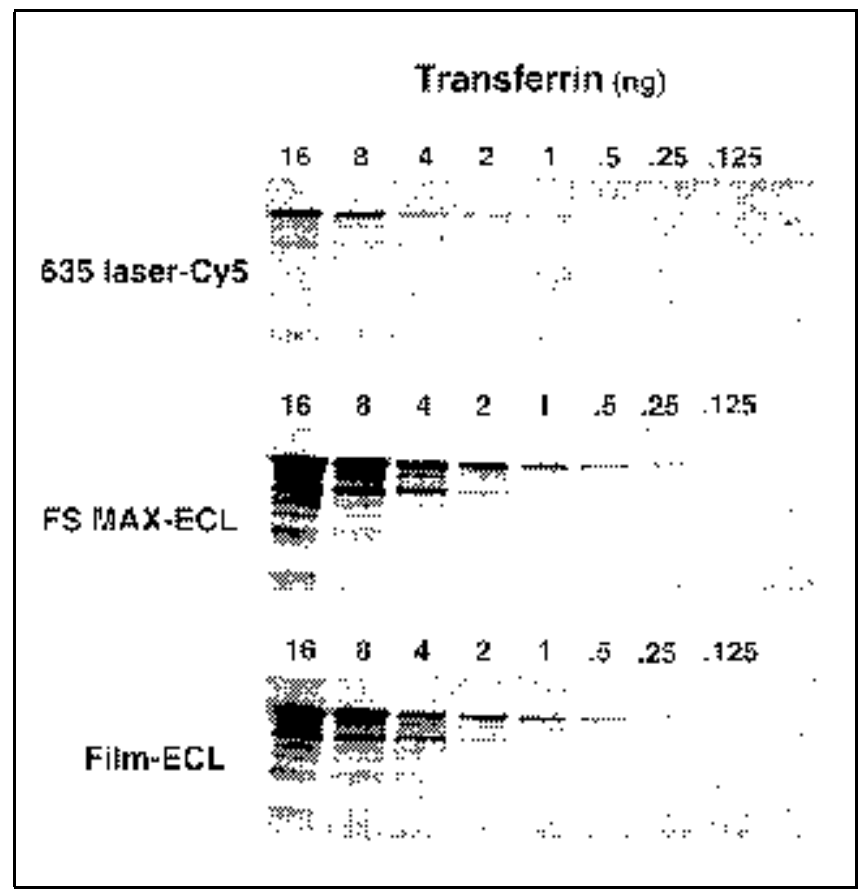

Figure 3. Comparision of limit of detection of transferrin protein on fluorescent versus chemiluminescent Western blots. A twofold serial dilution of human apo-transferrin protein was electrophoresed on 12\% SDS-polyacrylamide gels. Proteins were electroblotted onto supported nitrocellulose and the blots probed with rabbit anti-human transferrin followed by either Cy5- or HRP-conjugated goat anti-rabbit antibody. The Cy5-labeled blot was imaged on the Bio-Rad Molecular Imager FX using the 635-nm laser. The HRP-labeled blot was incubated with ECL and imaged first on the Bio-Rad Fluor-S MAX (FS MAX) for $1 \mathrm{~min}$, followed immediately by exposure to Xray film (Film) for $1 \mathrm{~min}$. The limit of detection using chemiluminescent detection is approximately twofold greater than using fluorescent detection. three serum proteins: albumin, transferrin and $\operatorname{IgG}$ (Figure 5). The fluorescent signals from transferrin relative to albumin are 3.7, 3.4 and 2.6 times higher than albumin in normal, patient 1 and patient 2, respectively, indicating little difference in the ratio of these two proteins in the three samples. In contrast, the level of IgG is dramatically increased relative to both transferrin and albumin in patient samples 1 and 2 compared to control. Quantitation of the ratio of fluorescent signal from IgG relative to albumin demonstrates that $\mathrm{IgG}$ is 5.1 times and 3.8 times higher in patients 1 and 2 and, relative to transferring, is 5.7 times and 5.5 times higher in patients 1 and 2, respectively, compared to control. The relative abundance of IgG is increased relative to control by 4.3 times and 4.8 times in patients 1 and 2, respectively, when measured by neph-

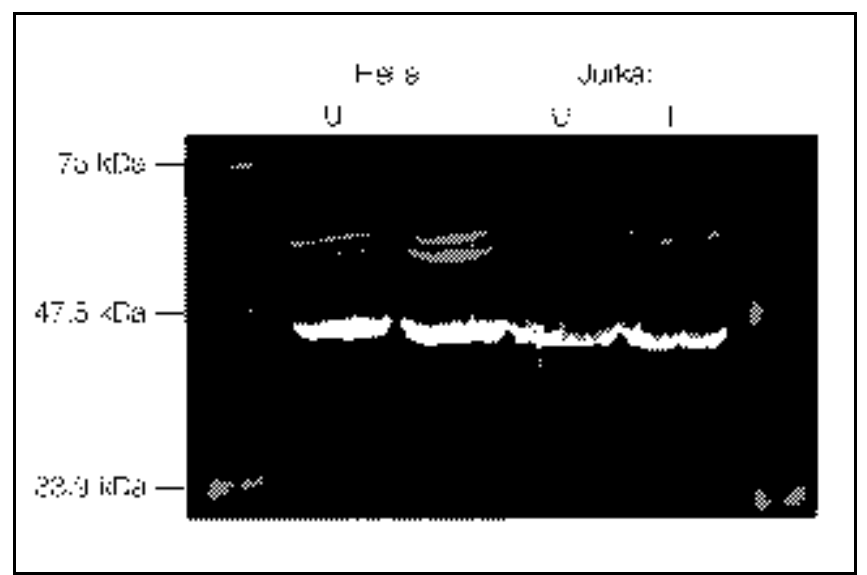

Figure 4. Three-color Western blot of proteins from cell lines before and after induction for apoptosis. Cell extracts from Jurkat and HeLa cells, induced and uninduced for apoptosis, were electrophoresed on a $12 \%$ polyacrylamide gel and the proteins were electroblotted onto supported nitrocellulose membrane. The blot was probed simultaneously with three probes directed against actin (FITC-labeled probe, green), caspase 8 (Cy3-labeled probe, orange), and PKA (Cy5-labeled probe, blue) as described in the Materials and Methods section. Three images were collected sequentially on the Bio-Rad Molecular Imager FX using each of the three lasers (488 nm, 532 $\mathrm{nm}$ and $635 \mathrm{~nm}$ ), and the images were merged using Confocal Assistant software. The level of caspase 8 (orange signal) increased relative to actin and PKA in apoptosis induced (I) compared to uninduced (U) cell cultures. Quantitative differences are noted in the text. 
elometry, the standard clinical test for determining IgG levels.

\section{DISCUSSION}

The data presented here illustrate the advantages and the limitations of using fluorescently labeled probes for Western blot detection. An important advantage is the ability to simultaneously probe multiple targets on a membrane. Simultaneous probing reduces the time and labor required for Western blot processing, a savings that is directly proportional to the number of probes used in a Western blot. Moreover, the required quantity of starting sample is less if one considers that only one blot has to be prepared.

We demonstrated multiplexed fluorescent Western detection using three probes labeled with FITC, Cy3 and Cy5. We were restricted to three probe/fluorochrome combinations primarily by the number of fluorochromes that can be discriminated following excitation with the three different laser lines available on the Molecular Imager FX scanner. Several other antibodies conjugated with different fluorochromes were tested (e.g., Alexa ${ }^{\mathrm{TM}}$ 488, 532 and 546, TRITC, Texas Red ${ }^{\circledR}$ and PBXL-1). In most cases, these other fluorescent probes showed limits of detection and linear range comparable to those demonstrated here. However, with the exceptions of Alexa 488 in place of FITC and TRITC in place of Cy3, we were not able to demonstrate the same level of spectral separation as the FITC, Cy3 and Cy5 combination. With software corrections for overlap or the use of additional laser lines, additional probes can be multiplexed on the same blot. For example, secondary antibodies are available that are labeled with AMCA (excitation maximum $350 \mathrm{~nm}$ and emission maximum $450 \mathrm{~nm}$ ), which is spectrally separated from FITC and the Cy dyes used here. However, a laser that efficiently excites this dye is not available with the FX system.

We have not investigated direct probing of the target proteins using fluorescently labeled primary antibodies. Others have demonstrated levels of detection similar to our results

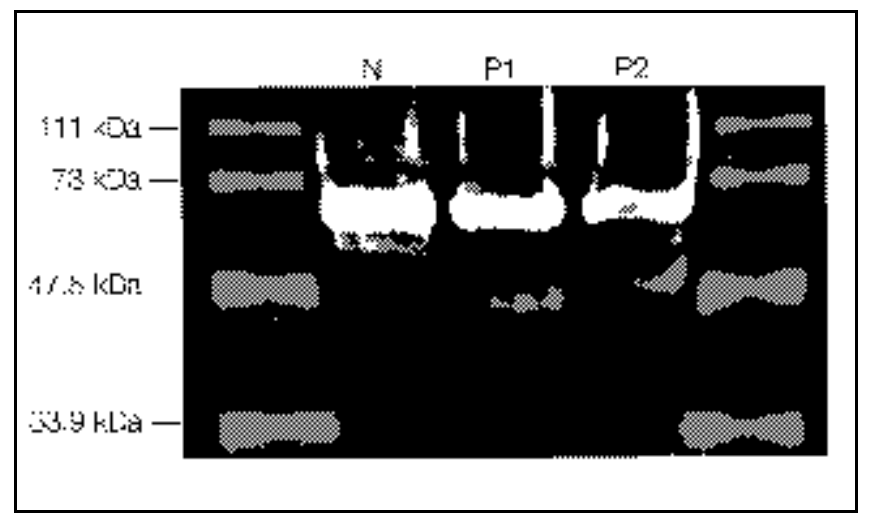

Figure 5. Three-color Western blot of human serum proteins. Equal amounts of human blood serum from three individuals were electrophoresed on a $12 \%$ polyacrylamide gel and electroblotted onto supported nitrocellulose membrane. The blot was probed with three antibodies directed against albumin (FITC-labeled probe, green), IgG (Cy3-labeled probe, orange) and transferrin (Cy5-labeled probe, blue). Three images were collected on the Bio-Rad Molecular Imager FX sequencially using three lasers (488, 532 and $635 \mathrm{~nm}$ ), and the images were merged using Confocal Assistant software. The level of IgG (orange signal) was elevated relative to albumin and transferrin in patients 1 and 2 (P1 and P2) compared to the normal control (N). Quantitative differences are noted in the text. 
using fluorescently labeled primary antibody probes (8). However, in a system using labeled primary antibodies, it is necessary to obtain a new fluorescent conjugate for each desired protein target. A staining system using fluorescent secondary antibodies allows much greater flexibility when designing antibody sets for multicolor Western blots. More specifically, one needs only a limited number of secondary antibodies that are complementary to the relatively small number of species of primary antibodies.

Our data show that the fluorescent signal from each of the three probes used here can be easily discriminated. Our examples using FITC, Cy3 and Cy5 show that the signal of each fluorochrome does not overlap unless there is a substantial difference in the protein concentration. For example, at least 1000-fold higher concentration in Cy3 or FITC target is needed for its signal to be equal to the Cy5 signal when imaging with the 635-nm laser. Even in the worse case, the $\mathrm{Cy} 3$ signal will be equal to the FITC signal when the Cy3-targeted protein concentration is 30 times higher than the FITC-targeted protein. However, with additional filter optimization, this overlap can be decreased to less than 1 in 1000 . Thus, greater than 1000-fold differences in protein concentrations can be accurately quantitated if needed.

Another advantage of using fluorescently labeled probes for Western blot detection is the range of quantitation com pared with other detection methods. The quantifiable linear range of the proteins using fluorescently labeled probes is 10 times greater than when using enhanced chemiluminescent substrates. The reason for this difference may be that fluorescent probes are detected directly and do not have the additional issue of enzyme/substrate kinetics associated with the horseradish peroxidase (HRP) interaction with its colorimetric or chemiluminescent substrates.

A previous report on the linear range of detection of a Western blot using a Cy5 reporter secondary antibody showed only a 20 -fold range in linear detection, not a 4000-fold range as shown here (3). However, in that report, it appears that only a 20-fold increase over the limit of detection was tested.

Despite the numerous advantages of using fluorescently labeled probes, they are limited in detection compared to chemiluminescent methods. Currently, the fluorescent probes tested exhibit two to four times less sensitivity than HRP-labeled probes with enhanced chemiluminescent substrates. This limitation may be largely due to the inherent fluorescence of the membranes. With both laser and UV excitation, the membrane fluoresces to some degree, but to a substantially higher extent with UV light. We find that the limit of detection of the same fluorescent probes tested here is decreased at least an additional 50-fold when the blots are epi-illuminated with UV light. When using chemiluminescence, the limit of detection is primarily due to the level of nonspecific binding of HRP antibodies to the membrane. This nonspecific binding generates luminescence when subjected to the chemiluminescent substrates. Another factor may be the brightness of the dye. We find that the limit of detection is improved about twofold when using PBXL-conjugated antibodies (data not shown). PBXLs are large aggregates of proteins containing multiple fluorescent moieties (8). Unfortunately, since the PBXL aggregates we tested excite and fluoresce at several different wavelengths, they cannot be used when multiplexed with other dyes to generate multiple resolved signals.

The utility of multiple protein detection and quantitation is demonstrated here in two applications. In the first model, we demonstrate up-regulation of a protein during apoptosis (5). The up-regulation of caspase 8, a serine protease in the Fas apoptotic pathway $(7,9,10)$, is demonstrated using two cell lines, Jurkat and HeLa, which were induced to undergo apoptosis using dexamethasone. Three-color Western blot analysis was carried out using antibodies directed against caspase 8, actin and PKA as constitutively expressed controls. In the second model system, three serum proteins (albumin, IgG and transferrin) are independently detected in several patient blood serum samples to demonstrate the relative increase in abundance of IgG relative to the other two proteins in a clinically relevant test system.

A survey of the literature shows that conclusions drawn from an increasing number of publications rely on demonstrating relative protein levels from cells grown under different conditions, from different cell types or in different disease states. The multiplex protein detection scheme described here should facilitate these and other studies in which more com parisons can be made faster and with better quantitation.

Note added in proof: The newest release of Quantity One software (version 4.2) supports image merging in three colors as shown in Figures 4 and 5.

\section{REFERENCES}

1.Bers, G. and D. Garfin. 1985. Protein and nucleic acid blotting and im munobiochemical detection. BioTechniques 3:276-288.

2.Bronstein, I., J.C. Voyta, O.J. Murphy, L. Bresnick and L.J. Kricka. 1992. Improved chemiluminescent Western blotting procedure. BioTechniques 12:748-752.

3.Fradelizi, J., E. Friederich, M.C. Beckerle and R.M. Golsteyn. 1998. Quantitative measurement of proteins by Western blotting with Cy5-coupled secondary antibodies. BioTechniques 26:484-494.

4.Kaufmann, S.H., C.M. Ewing and J.H. Shaper. 1987. The erasable Western blot. Anal. Chem. 161:89-95.

5.Kerr, J.F.R., A.H. Wyllie and A.R. Curie. 1972. Apoptosis: a basic biological phenomenon with wide-ranging implications in tissue kinetics. Br. J. Cancer 26:239-257.

6.Kricka, L.J. 1991. Chemiluminescent and bioluminescent techniques. Clin. Chem. 37:1472-1481.

7.Majno, G. and J. Joris. 1995. Apoptosis, oncosis and necrosis. An overview of cell death. Am. J. Pathol. 146:3-16.

8.Morseman, J.P., M.W. Moss, S.J. Zoha and F.C.T. Allnutt. 1999. PBXL-1: a new fluorochrome applied to detection of proteins on mem branes. BioTechniques 26:559-563.

9.Muzio, M., A.M. Chinnaiyan, F.C. Kischkel, K. O'Rourke, A. Shevchenko, J. Ni, C. Scaffidi, P.H. Krammer et al. 1996. FLICE, a novel FADD-homologous ICE/Ced-3-like protease, is recruited to the CD95 (Fas/APO-1) death-inducing signaling complex. Cell 85:817-827.

10.Nagata, S. and P. Goldstein. 1995. The Fas death factor. Science 267:1449-1456.

11.Poor, M.L., P.F. Santa and G.S. Sittampalam. 1988. Visualization of multiple protein bands on the same nitrocellulose membrane by double immunoblotting. Anal. Biochem. 175:191-195.

12.Sambrook, J., E.F. Fritsch and T. Maniatis. 1989. Molecular Cloning: A Laboratory Manual. CSH Laboratory Press, Cold Spring Harbor, NY.

13.Steffen, W. and R.W. Linek. 1989. Multiple immunoblot: a sensitive technique to stain proteins and detect multiple antigens on a single twodimensional replica. Electrophoresis 10:714-718.

14.Thorpe, G.H.G., L.J. Kricka, S.B. Moseley and T.P. Whitehead. 1985 Phenols as enhancers of the chemiluminescent horseradish peroxidase-luminol-hydrogen peroxide reaction: application in luminescence-monitored enzyme immunoassays. Clin. Chem. 31:1335-1341.

Address correspondence to Dr. Jeffrey C. Gingrich, Imaging Products Division, Bio-Rad Laboratories, 2000 Alfred Nobel Drive, Hercules, CA 94704,USA.e-mail: jgingrich@ bio-rad.com 